Copyright by the Acoustical Society of America. Chaudhry, Z., Lalande, F., \& Rogers, C. A. (1995). Modeling of induced strain actuation of shell structures. Journal of the Acoustical Society of America, 97(5), 2872-2877. doi: 10.1121/1.411853

\title{
Modeling of induced strain actuation of shell structures
}

\author{
Zaffir Chaudhry, Frederic Lalande, and Craig A. Rogers \\ Center for Intelligent Material Systems and Structures, Virginia Polytechnic Institute and State University, \\ Blacksburg, Virginia 24061-0261
}

(Received 25 May 1994; accepted for publication 17 November 1994)

\begin{abstract}
Based on the thin-shell Donnell theory, a model to represent the action of discrete induced strain actuator patches symmetrically bonded to the surface of a circular cylindrical shell has been developed. The model provides estimates of the bending curvatures due to the out-of-phase actuation and the in-plane strains due to the in-phase actuation of the bonded actuator patches. The magnitudes of the induced curvature and the in-plane strain are found to be identical to those of plates; however, due to the strain-displacement relations in cylindrical coordinates, the in-plane and out-of-plane displacements are coupled. Expressions for the equivalent forces and moments that represent the action of the actuator patches have been developed. Due to the curvature of the shell, the representation of the in-phase actuation with an equivalent in-plane line force applied along the edge of the actuator results in the application of erroneous rigid-body transverse forces. To avoid these rigid body forces, a method to represent the in-phase actuation with a system of self-equilibrating forces is proposed. The action of the actuator is then represented by an equivalent in-plane force and a transverse distributed pressure applied in the region of the actuator patch. Finite element verification of the proposed model is presented. The displacements due to the actual actuator actuation are compared with the proposed model, and very good agreement is found.
\end{abstract}

PACS numbers: $43.40 . E y, 43.40 . T m$

\section{INTRODUCTION}

In recent years there has been a great surge of interest in research on shape, vibration, and acoustic control of structures with induced strain actuators like piezoelectric materials and shape memory alloys. What distinguishes induced strain actuators from conventional hydraulic and electrical actuators, and makes them especially attractive for smart structures, is their ability to change their dimensions and properties without utilizing any moving parts. These actuator materials contract and expand just like the muscles in the human body. When integrated into a structure (either through embedding or through surface-bonding), they apply localized strains and directly influence the extensional and bending responses of the structural elements. Because of the absence of mechanical parts they can be easily integrated into the base structure. Integration within the structure ensures an overall force equilibrium between the forcing actuator and the deforming structure, thus precluding any rigid body forces and torques.

Induced strain actuators, like piezoelectric materials when bonded to the surface of a structure, generate a set of forces which are concentrated close to the edges of the actuator. Therefore, their action is often represented by line moments or forces applied along the periphery of the actuator. This representation simplifies analysis because the structure does not have to be discretized (to represent the nonuniform structural properties in the regions of the patches) and global structural equations can be solved with the actuator forces appearing as discretely applied external forces. This analysis method, although approximate (approximate because the actuator mass and stiffness are not represented and actuator forces derived from static and stress-free boundary conditions are used), gives reasonably accurate results for small and thin patches of actuators. Also, for the case of straight structural members like beams and plates, it does not pose any problems such as the equivalent actuator forces producing rigid-body motion. For shell-type structures, however, due to their curvature, this simple representation is not appropriate for the case of in-phase actuation. In-phase actuation refers to the case when the two actuators bonded to the top and bottom surface of the shell are activated to produce strains in the same direction. Because the circumferential forces used to represent the action of the actuator are not colinear they produce rigid-body transverse forces on the shell. Thus certain special modifications must be made to such a representation scheme to accommodate the special characteristics of the shell structures. The modification proposed and verified in this paper is the application of a uniform transverse pressure across the footprint of the actuator.

To date, a number of models to represent the action of actuators on beams and plates have been proposed. ${ }^{1-5}$ For shells, the only models that have been developed are based on layered shell theory, i.e., the analytical model assumes that the induced strain actuator material comprises a total, distinct layer of the shell. ${ }^{6,7}$ In the work that has been reported on vibration and acoustic control of shells using piezoelectric actuators, plate models are often adapted to shells. ${ }^{8,9}$ At first sight, this adaptation seems perfectly reasonable because the shell is thin and $r / h$ is large. This does, however, pose a problem for the representation of in-phase actuation forces because the actuator forces are no longer colinear as in the case of beams and plates, and as stated earlier, this results in a rigid-body force being applied to the shell (see Fig. 1). If this is not recognized, then the action of in-phase actuation of even a small actuator patch will result in an erroneous response and can lead to a totally wrong 


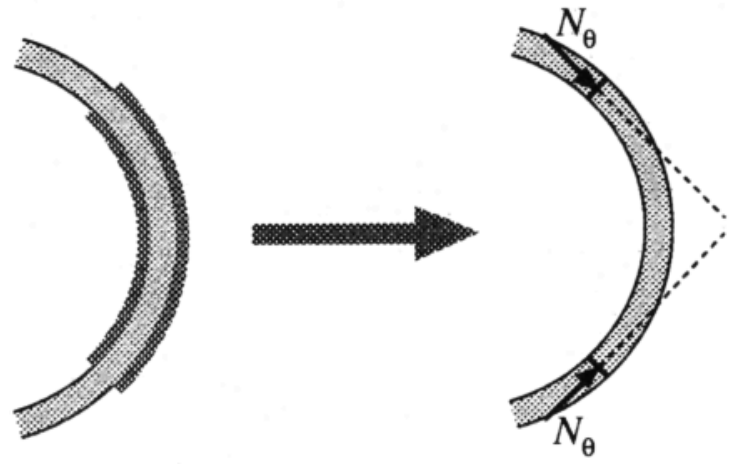

FIG. 1. Nonequilibrium of discrete tangential forces in shell structures.

solution. In a recent model for curved piezoelectric actuators, Sonti and Jones ${ }^{10}$ also recognized this fact and showed the necessity of including a uniform transverse pressure, in addition to the axial and tangential forces, to correctly represent the action of in-phase actuation.

A model has been developed to compute the equivalent forces and moments applied by a pair of symmetrically bonded actuator pairs, and is described here. This is followed by a discussion of the special considerations for shell-type structures. The development of the model is similar to the work of Crawley and Lazarus ${ }^{1}$ for plates, and relies on classical lamination theory (CLT).

\section{MODEL FORMULATION}

A model describing the interaction between surfacebonded actuators and a circular cylindrical shell has been developed based on Donnell's theory. For completeness a short derivation of the accompanying equations of motion is also presented. Consider a thin circular cylindrical shell, as shown in Fig. 2. We start with the following Donnell's assumptions $(1+z / R=1, r \rightarrow R)$ for the kinematics of deformation:

$$
\begin{aligned}
& u(x, \theta, z)=u^{0}(x, \theta)-z \frac{\partial w^{0}}{\partial x}, \\
& \nu(x, \theta, z)=\nu^{0}(x, \theta)-z \frac{\partial w^{0}}{R \partial \theta}
\end{aligned}
$$

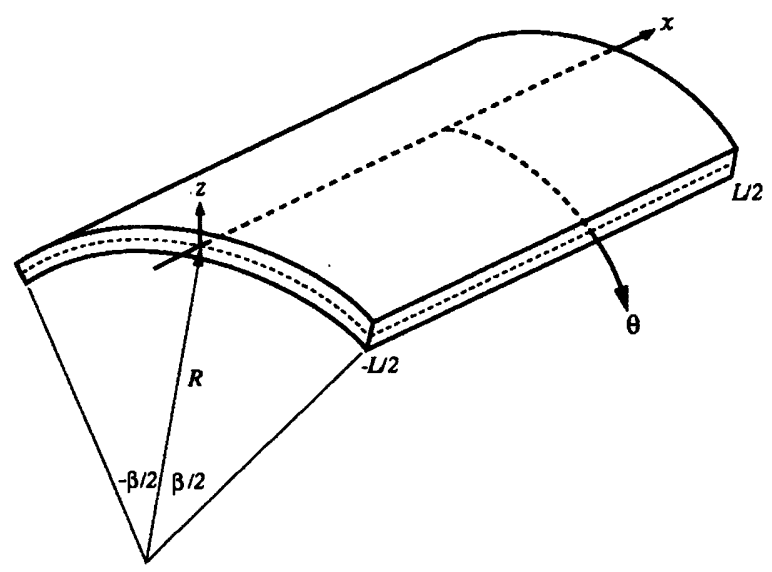

FIG. 2. Thin circular cylindrical shell coordinate system.

$$
w(x, \theta, z)=w^{0}(x, \theta) .
$$

In the above, $z$ is the local thickness coordinate, measured from the middle surface of the shell. The superscript zero denotes displacements of the cylindrical panel's middle surface. The pertinent strain-displacement relations in cylindrical coordinates are as follows:

$$
\begin{aligned}
& \epsilon_{x}=\frac{\partial u}{\partial x}=\epsilon_{x}^{0}+z \kappa_{x}, \quad \epsilon_{x}^{0}=\frac{\partial u^{0}}{\partial x}, \quad \kappa_{x}=-\frac{\partial^{2} w^{0}}{\partial x^{2}}, \\
& \epsilon_{\theta}=\frac{\partial \nu}{R \partial \theta}+\frac{w}{R}=\epsilon_{\theta}^{0}+z \kappa_{\theta}, \quad \epsilon_{\theta}^{0}=\frac{\partial \nu^{0}}{R \partial \theta}+\frac{w^{0}}{R},
\end{aligned}
$$

$$
\kappa_{\theta}=-\frac{\partial^{2} w^{0}}{R^{2} \partial \theta^{2}}
$$

and

$$
\begin{aligned}
& \gamma_{x \theta}=\frac{\partial \nu}{\partial x}+\frac{\partial u}{R \partial \theta}=\gamma_{x \theta}^{0}+z \kappa_{x \theta}, \quad \gamma_{x \theta}^{0}=\frac{\partial \nu^{0}}{\partial x}+\frac{\partial u^{0}}{R \partial \theta}, \\
& \kappa_{x \theta}=-2 \frac{\partial^{2} w^{0}}{\partial x R \partial \theta} .
\end{aligned}
$$

The resultant force-strain relations for a layered composite laminate are given by ${ }^{11}$

$$
\left\{\begin{array}{l}
N \\
M
\end{array}\right\}=\left[\begin{array}{ll}
A & B \\
B & D
\end{array}\right]\left\{\begin{array}{c}
\epsilon^{0} \\
\kappa
\end{array}\right\}-\left\{\begin{array}{l}
N_{\Lambda} \\
M_{\Lambda}
\end{array}\right\},
$$

where

$$
\begin{aligned}
& \left\{N_{\Lambda}\right\}=\int[\bar{Q}]\{\Lambda\} d z, \\
& \left\{M_{\Lambda}\right\}=\int[\bar{Q}]\{\Lambda\} z d z
\end{aligned}
$$

are the equivalent of thermal forces in CLT. Note that $\Lambda$ is the free induced strain developed in the actuator in response to an applied voltage $\left(\Lambda_{x}=E d_{3 x}=V d_{3 x} / t_{a}\right)$. The three governing equilibrium equations are:

$$
\begin{aligned}
& \frac{\partial N_{x}}{\partial x}+\frac{\partial N_{x \theta}}{R \partial \theta}-m \ddot{u}=0, \\
& \frac{\partial N_{x \theta}}{\partial x}+\frac{\partial N_{\theta}}{R \partial \theta}-m \ddot{\nu}=0,
\end{aligned}
$$

and

$$
\frac{\partial^{2} M_{x}}{\partial x^{2}}+2 \frac{\partial^{2} M_{x \theta}}{\partial x R \partial \theta}+\frac{\partial^{2} M_{\theta}}{R^{2} \partial \theta^{2}}-\frac{N_{\theta}}{R}-m \ddot{w}=q,
$$

with boundary conditions at $x= \pm L / 2$ and $\theta= \pm \beta / 2$. For an unconstrained symmetric panel with no externally applied forces or moments, i.e., $N, M=0$, Eq. (7) reduces to

$$
\left\{\begin{array}{c}
\epsilon^{0} \\
\kappa
\end{array}\right\}=\left[\begin{array}{ll}
A & B \\
B & D
\end{array}\right]^{-1}\left\{\begin{array}{l}
N_{\Lambda} \\
M_{\Lambda}
\end{array}\right\} .
$$

For a symmetric shell $(B=0)$, the above equations are uncoupled, and

$$
\left\{\epsilon^{0}\right\}=[A]^{-1}\left\{N_{\Lambda}\right\},
$$




$$
\{\kappa\}=[D]^{-1}\left\{M_{\Lambda}\right\} .
$$

To obtain simplified expressions for the induced curvature, we assume that the shell is isotropic and has the same Poisson's ratio as the actuator. For such a case, the $[A]$ and $[D]$ matrices reduce to:

$$
\begin{aligned}
{[A]=} & \frac{E_{s} t_{s}+2 E_{a} t_{a}}{\left(1-\nu^{2}\right)}\left[\begin{array}{ccc}
1 & \nu & 0 \\
\nu & 1 & 0 \\
0 & 0 & (1-\nu) / 2
\end{array}\right], \\
{[D]=} & \left\{\frac{E_{s} t_{s}^{3}}{12\left(1-\nu^{2}\right)}+\frac{2}{3} \frac{E_{a}}{\left(1-\nu^{2}\right)}\left[\left(\frac{t_{s}}{2}+t_{a}\right)^{3}-\left(\frac{t_{s}}{2}\right)^{3}\right]\right\} \\
& \times\left[\begin{array}{ccc}
1 & \nu & 0 \\
\nu & 1 & 0 \\
0 & 0 & (1-\nu) / 2
\end{array}\right] .
\end{aligned}
$$

Rewriting in a more convenient way,

$$
\begin{aligned}
{[A]=} & \frac{E_{a} t_{a}(2+\psi)}{\left(1-\nu^{2}\right)}\left[\begin{array}{ccc}
1 & \nu & 0 \\
\nu & 1 & 0 \\
0 & 0 & (1-\nu) / 2
\end{array}\right], \\
{[D]=} & \frac{t_{s}^{2}}{12} \frac{E_{a} t_{a}}{\left(1-\nu^{2}\right)}\left[6+\psi+\frac{12}{T}+\frac{8}{T^{2}}\right] \\
& \times\left[\begin{array}{ccc}
1 & \nu & 0 \\
\nu & 1 & 0 \\
0 & 0 & (1-\nu) / 2
\end{array}\right] .
\end{aligned}
$$

With these assumptions, the following expression for the induced bending strains due to the out-of-phase actuation is obtained from Eq. (15) $\left(t_{s}=\right.$ shell thickness, $t_{a}=$ actuator thickness):

$$
\left\{\begin{array}{c}
\kappa_{x} \\
\kappa_{\theta} \\
\kappa_{x \theta}
\end{array}\right\}=\frac{6(1+1 / T)\left(2 / t_{s}\right)}{6+\psi+8 / T^{2}+12 / T}\left\{\begin{array}{l}
1 \\
1 \\
0
\end{array}\right\} \Lambda,
$$

where

$$
\psi=\frac{E_{s} t_{s}^{\prime}}{E_{a} t_{a}}, \quad T=\frac{t_{s}}{t_{a}},
$$

and for in-phase actuation, the following expression for induced middle-surface strains is obtained from Eq. (14):

$$
\left\{\begin{array}{c}
\epsilon_{x}^{0} \\
\epsilon_{\theta}^{0} \\
\epsilon_{x \theta}^{0}
\end{array}\right\}=\frac{2}{2+\psi}\left\{\begin{array}{l}
1 \\
1 \\
0
\end{array}\right\} \Lambda .
$$

The above expressions for the middle-surface strains for the case of in-phase actuation and the bending strains for the case of out-of-phase actuation are the same as those obtained for plates; however, the circumferential strain is now coupled with the out-of-plane displacement [Eq. (5)].

To solve a plate or a shell problem, an expression for a set of equivalent forces is often developed. These forces, when applied along the edges of the footprint of the actuator, on the structure result in the same degree of bending strains and in-plane middle-surface strains as those obtained from

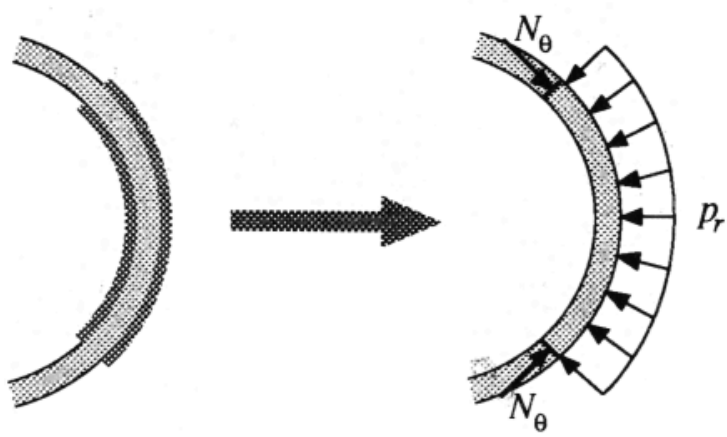

FIG. 3. Adequate equivalent loading to maintain equilibrium.

Eqs. (20) and (22). The equivalent forces and moments are obtained by substituting the bending strains and the in-plane strains from Eqs. (20) and (22) into Eq. (7), with $N_{\Lambda}$ and $M_{\Lambda}$ set to zero. For out-of-phase actuation, the equivalent moments are found to be:

$$
\left\{\begin{array}{c}
M_{x} \\
M_{\theta} \\
M_{x \theta}
\end{array}\right\}=\frac{E_{s} t_{s}^{2}}{1-\nu} \frac{(1+1 / T)}{6+\psi+8 / T^{2}+12 / T}\left\{\begin{array}{l}
1 \\
1 \\
0
\end{array}\right\} \Lambda,
$$

and for in-phase actuation, the following expression for the equivalent axial and tangential force is obtained:

$$
\left\{\begin{array}{c}
N_{x} \\
N_{\theta} \\
N_{x \theta}
\end{array}\right\}=\frac{E_{s} t_{s}}{1-\nu} \frac{2}{2+\psi}\left\{\begin{array}{l}
1 \\
1 \\
0
\end{array}\right\} \Lambda
$$

The tangential force obtained from the above equation, when applied to the shell along the two circumferential edges of the actuator will not be colinear, due to the curvature of the shell, and will have an erroneous component resulting in a rigid body mode. This situation has already been illustrated in Fig. 1. This occurs due to the simplifying assumptions in the above formulation. In actuality, a set of self-equilibrating stresses are developed between the shell and the bonded actuator, and there is no rigid body force developed.

To remedy this situation, we have proposed the application of an equilibrating uniform radial pressure applied across the footprint of the actuator. The magnitude of the uniform pressure is obtained from simple statics and is equal to $-N_{\theta} / R$ (Fig. 3). This set of forces, i.e., an equivalent tangential force given by Eq. (24) and a uniform radial pressure, now provide a convenient means of representing the action of surface-bonded actuators that are actuated in-phase. To verify whether this set of forces results in approximately the same displacement field as a true actuator bonded to a cylindrical shell (a comparison with a finite element model) a description of which follows, is made.

\section{FINITE ELEMENT MODELING AND VERIFICATION}

Two finite element models have been constructed to verify the equivalent loading scheme. A 6-in.-radius, 0.032in.-thick, and 1-in.-deep ring with piezoelectric actuators $1 / 6$ of the ring thickness and covering an arc $10^{\circ}$ long $(\beta)$ have been used. Making use of symmetry, only the top half needs to be modeled. The first model, shown in Fig. 4(a), consists 

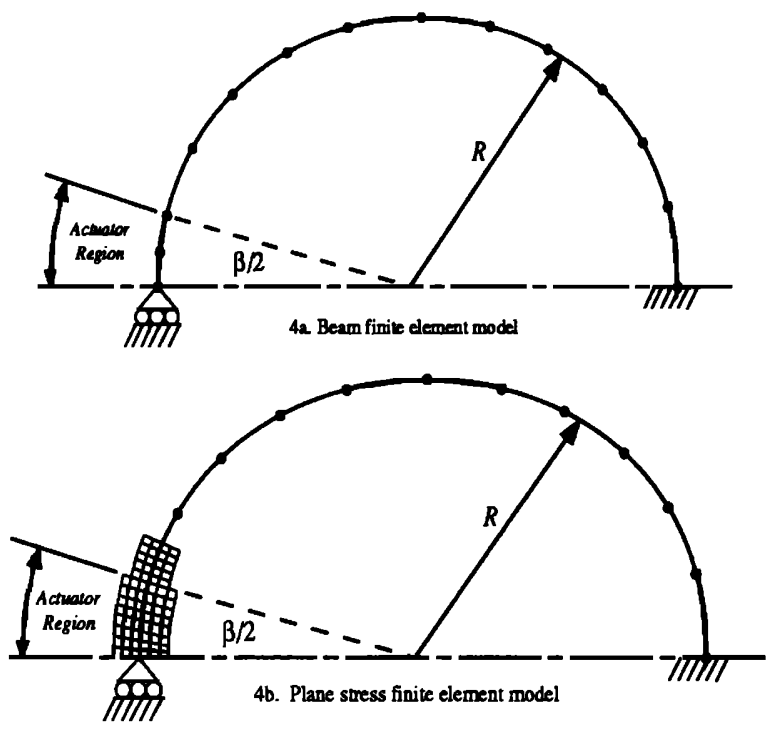

FIG. 4. Finite element models used to verify the theoretical model.

of beam elements. First, the actuation is simulated by specifying a coefficient of thermal expansion for the elements in the actuator region and then applying a known temperature to the model. Second, an equivalent self-equilibrating load, i.e., pressure and tangential force, corresponding to the temperature, is applied [Eqs. (23) and (24)]. The radial and tangential displacements obtained from the above analysis are identical and therefore not shown. It must be noted that the pressure loading must be transformed to nodal forces only (lumped loading). The lumped loading is often better for flat elements representing a curved surface. ${ }^{12}$ The second finite element model uses plane stress elements in the actuator region to include the actuator's stiffness and uses beam elements for the rest of the shell [Fig. 4(b)]. A rigid element connecting the five nodes at the end of the plane stress element region is introduced. A constraint equation is then used to ensure the continuity in the rotations between the beam and plane stress elements. Again, thermal expansion is used to simulate the static action of the actuators on the shell.

The radial and tangential displacements are shown in Fig. 5. Discrepancies between the equivalent loading model and the plane stress finite element model exist since no assumptions about the actuator stiffness or about the equivalent loading are made in the latter model. The differences are however greater in the actuator region due to the added structural stiffness of the actuators. Even though displacement differences are present, the plane stress finite element model validates the derived model since it gives results of the same order of magnitude with similar deformed shapes as opposed to when only point tangential forces (without a transverse pressure) are used. The deformed shape of the analytical model and the plane stress finite element model are shown in Fig. 6. Also shown in Fig. 5 are the displacements of the same ring if only discrete tangential forces are applied (without transverse pressure). This model using only tangential forces does not satisfy the ring's self-equilibrium. Major displacement discrepancies between the proposed equivalent loading model and the case using only tangential forces are observed both in shape and magnitude. Using the tangential forces alone over predicts the displacements by a factor of up to 1000 , as seen on the right vertical axis of Fig. 5 (note that the scales of the two vertical axis are different). Also, a re-

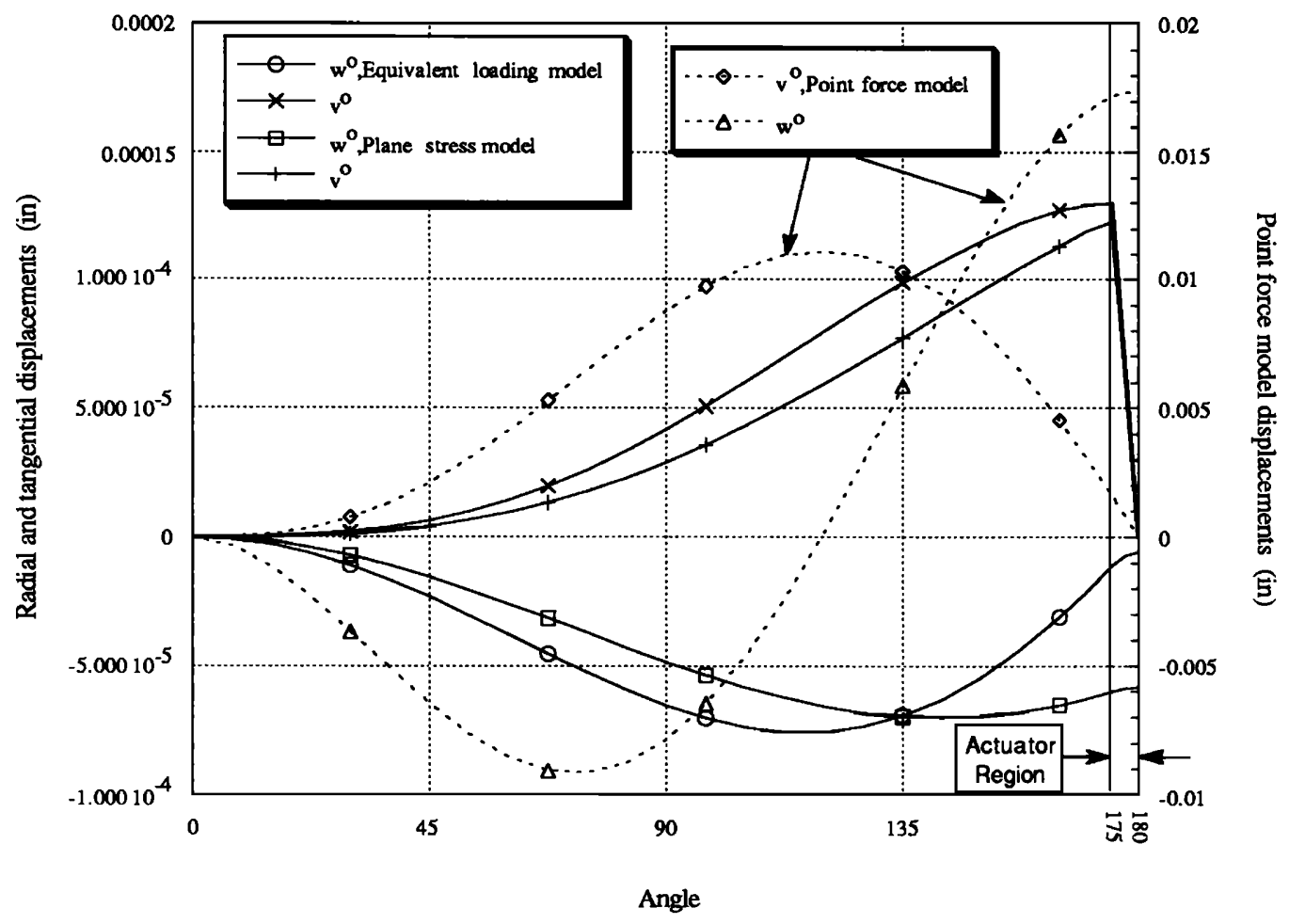

FIG. 5. Comparison of the displacements predicted by the proposed self-equilibrating equivalent forces, the plane stress finite element model, and the tangential force alone (no pressure). 


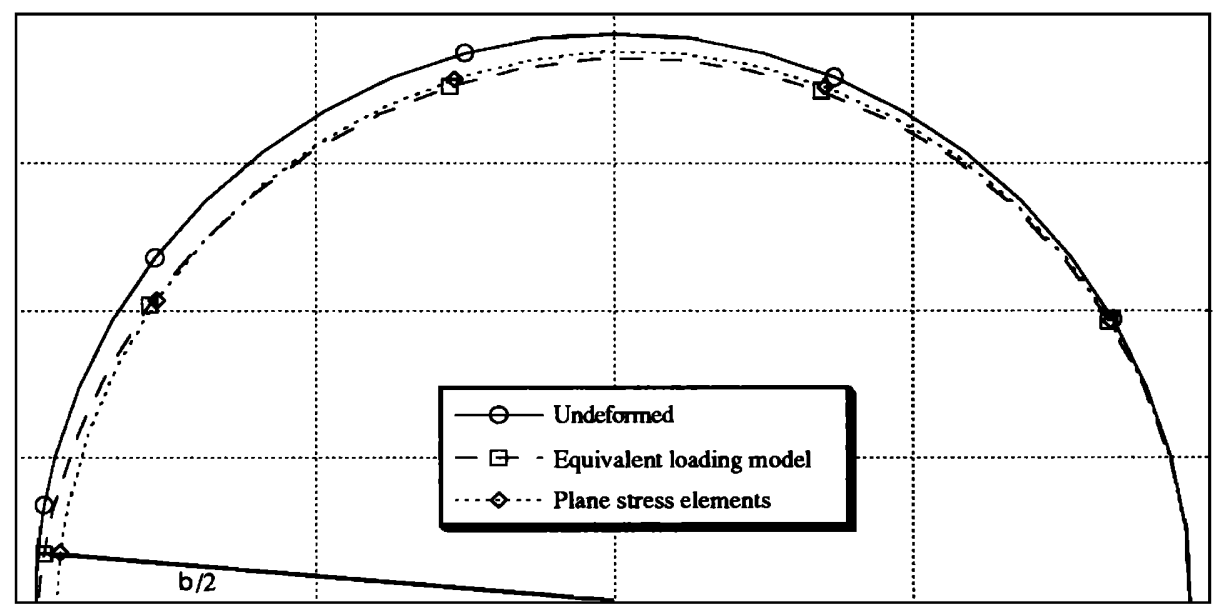

FIG. 6. Deformed shape of the ring using the self-equilibrium loading and the plane stress elements.

action force in the $x$ direction at the clamped boundary is present if uniform pressure is not applied. This reaction force should not be present since the actual ring with bonded actuators is in self-equilibrium. Adequate equivalent loading did not show any reaction force in the $x$ direction at the clamped boundary.

From the finite plane stress element model shown in Fig. 4(b), it is possible to justify the use of a uniform radial pressure on the actuator footprint to maintain the self-equilibrium of the ring. Figure 7 shows the radial stress distribution through the thickness of the actuator and the ring. This stress distribution is virtually constant over the whole actuator region, except at the ends of the actuator, which validates the use of a uniform radial pressure in the analytical model. With the actuators removed, an equilibrating radial pressure applied on both sides of the shell is necessary to produce the same stress distribution on the shell surface $(z / h=1,-1)$ shown in Fig. 7. Because the interest is in global shell deformations, the equilibrating pressure can be applied on one side only or on the shell midplane, since it will produce the same shell response.

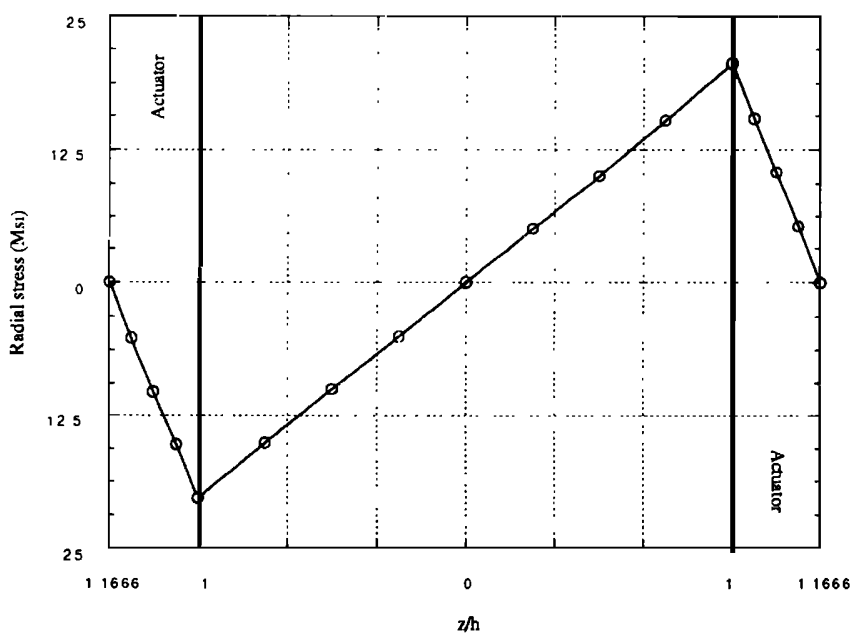

FIG. 7. Radial stress distribution through the thickness in the actuator region.

\section{CONCLUSIONS}

In this paper, a model to represent the action of discrete induced strain actuator patches bonded to the surface of a circular cylindrical shell is developed based on Donnell's theory. Expressions to represent the actuator forces and moments have been developed for shells and are found to be the same as those obtained for plates. However, this equivalent set of forces and moments produces a rigid body mode resulting from the no-colinearity of the tangential forces due to the shell curvature. To avoid this rigid body mode, uniform pressure is applied in the region of the actuator patch. This solution method is verified using finite element modeling and showed very good agreement.

\section{ACKNOWLEDGMENTS}

The authors would like to acknowledge the funding support of the Office of Naval Research, Grant No. ONR N00014-92-J-1170.

${ }^{1}$ E. F. Crawley and K. B. Lazarus, "Induced Strain Actuation of Isotropic and Anisotropic Plates," AIAA J. 29(6), 944-951 (1989).

${ }^{2}$ C. K. Lee, "Theory of laminated piezoelectric plates for the design of distributed sensors/actuators. Part I: Governing equations and reciprocal relationships," J. Acoust. Soc. Am. 87, 1144-1158 (1990).

${ }^{3}$ E. F. Crawley and E. H. Anderson, "Detailed Models of Piezoceramic Actuation of Beams," J. Intell. Mater. Syst. Struct. 1, 4-25 (1990).

${ }^{4}$ B. T. Wang and C. A. Rogers, "Modeling of Finite-Length Spatially Distributed Induced Strain Actuators for Laminate Beams and Plates," J. Intell. Mater. Syst. Struct. 2, 38-58 (1991).

${ }^{5}$ E. K. Dimitriadis, C. R. Fuller, and C. A. Rogers, "Piezoelectric Actuators for Distributed Vibration Excitation of Thin Plates," J. Vib. Acoust. 113, 100-107 (1991).

${ }^{6}$ H. S. Tzou, "Theoretical Development of a Layered Thin Shell with Internal Distributed Controllers," Failure Reliability Conference, Montreal, Canada (1989).

${ }^{7}$ J. Jia and C. A. Rogers, "Formulation of a Laminated Shell Theory Incorporating Embedded Distributed Actuators," J. Mech. Design 112, 596604 (1990).

${ }^{8}$ V. Sonti and J. D. Jones, "Active Vibration Control of Thin Cylindrical Shells Using Piezoelectric Actuators," in Proceedings: Recent Advances in Active Control of Sound and Vibration, Blacksburg, VA (Technomic, Lancaster, PA, 1991), pp. 3-26. 
${ }^{9}$ H. C. Lester and S. Lefebvre, "Piezoelectric Actuator Models for Active Vibration Control of Cylinders," in Proceedings: Recent Advances in Active Control of Sound and Vibration, Blacksburg, VA (Technomic, Lancaster, PA, 1991), pp. 3-26.

${ }^{10}$ V. R. Sonti and J. D. Jones, "Curved Piezo-Actuator Models for Active
Vibration Control of Cylindrical Shells," J. Acoust. Soc. Am. 93, 2352 (A) (1993).

${ }^{11}$ R. M. Jones, Mechanics of Composite Materials (Scripta, Washington, DC, 1975).

${ }^{12}$ G. J. De Salvo and J. A. Swanson, ANSYS User's Manual (Swanson Analysis Systems, Houston, PA, 1979). 